\title{
Representación social de la diabetes tipo 2 en mujeres bajo la teoría del núcleo central*
}

\author{
Social Representation of the Diabetes Type 2 in Women according to the Central Core Theory Representação social \\ da diabete tipo $2 \mathrm{em}$ mulheres sob a teoria do núcleo central
}

Ruth Angélica Landa Rivera ${ }^{a}$

Universidad Veracruzana, México

angelica8@hotmail.com

ORCID: http://orcid.org/0000-0002-1018-3571

Lubia del Carmen Castillo Arcos

Universidad Autónoma del Carmen, México

ORCID: http://orcid.org/0000-0002-4368-4735

Nicolás Padilla Raygoza

Universidad de Guanajuato, México

ORCID: http://orcid.org/0000-0002-2109-5163
DOI: https://doi.org/10.11144/Javeriana.ie21-1.rsdm Redalyc: http://www.redalyc.org/articulo.oa? id $=145257605007$

Fecha de recepción: 23 Abril 2016 Fecha de aprobación: 11 Octubre 2017

\section{Resumen:}

Introducción: La diabetes como enfermedad crónica es un evento catastrófico que origina comportamientos negativos hacia la adherencia terapéutica, lo que hace necesario usar teorías cognoscitivas y sociales para reorientar los cuidados de enfermería. Objetivo: Dar respuesta a las preguntas orientadoras de la investigación: ¿̨cuál es la representación social que la mujer tiene sobre la diabetes tipo 2? ¿Qué elementos integran el núcleo central y los sistemas periféricos de la representación social? Método: Revisión integrativa utilizando como guía el método prisma, de artículos cuyos descriptores primarios fueran "representaciones sociales", "diabetes tipo 2" y "mujeres" y secundarios "pacientes con diabetes tipo 2". La búsqueda se realizó en bases de datos informatizadas: Web of Science, Medline, PubMed, SciELO, LILACS, Bireme y CUIDEN. Resultados: Los hallazgos señalan que el fenómeno de la diabetes dio origen a una representación social irreversible. Este sistema de informaciones, actitudes y creencias se organizan y estructuran alrededor del padecimiento, alimentación, ejercicio y medicación, lo que dificulta la identidad social y, probablemente, la adherencia terapéutica a la enfermedad. Conclusión: El identificar la estructura de la representación social y elementos que integran el núcleo central les permite a los profesionales de la salud planear intervenciones que favorezcan la identidad social y la adherencia terapéutica de las mujeres en la diabetes tipo 2 . Palabras clave: diabetes tipo 2, representación social, mujer, adherencia.

\section{Abstract:}

Introduction: As a chronic disease, diabetes is a disastrous event bringing about negative behaviors regarding the treatment adherence, which makes necessary the use of cognitive and social theories in order to redirect the nursing care. Objective: To answer the guiding research questions: What is the social representation that women have about Diabetes Type 2? What elements form the central core and the peripheral systems of the social representation? Method: This is a comprehensive review using the Prism Method as a guide. It includes articles whose primary descriptors are "social representations", "Diabetes Type 2" and "women"; and the secondary descriptor "type 2 diabetes patients". The search was carried out in systematized databases: Web of Science, Medline, PubMed, SciELO, LILACS, Bireme, and CUIDEN. Results: The findings indicate that diabetes phenomenon gave rise to an irreversible social representation. This system of data, attitudes and beliefs is organized and structured around the suffering, feeding, exercise and medication, which makes difficult to ascertain the social identity and, probably, the treatment adherence specific to this disease. Conclusion: To identify the structure of the social representation and elements forming the central core allows the health professionals to plan interventions intended to favor the social identity and treatment adherence among women with diabetes type 2.

Keywords: Diabetes type 2, social representation, woman, adherence.

$$
\text { a Autor de correspondencia: lruth_angelica8@hotmail.com }
$$




\section{Resumo:}

Introdução: $\mathrm{O}$ diabete como uma doença crónica é um evento catastrófico que origina comportamentos negativos para a adesão terapêutica, o que faz preciso usar teorias cognoscitivas e sociais para reorientar os cuidados de enfermagem. Objetivo: Dar resposta às perguntas norteadoras da pesquisa: qual a representação social que a mulher possue sobre o diabete tipo 2? Quais elementos compõem o núcleo central e os sistemas periféricos da representação social? Método: Revisão integrativa utilizando como guia o método prisma, de artigos cujos descritores primários foram "representações sociais", "diabete tipo 2" e "mulheres" e secundários "pacientes com diabete tipo 2". A busca foi realizada em bases de dados informatizadas: Web of Science, Medline, PubMed, SciELO, LILACS, Bireme e CUIDEN. Resultados: Os achados apontam que o fenômeno da diabete deu origem a uma representação social irreversível. Esse sistema de informações, atitudes e crenças é organizado e estruturado em torno do padecimento, alimentação, exercício e medicação, o que dificulta a identidade social e, provavelmente, a adesão terapêutica à doença. Conclusão: Identificar a estrutura da representação social e elementos que integram o núcleo central permite os pro fissionais de a saúde planejarem intervenções favorecendo a identidade social e a adesão terapêutica das mulheres em diabetes tipo 2.

Palavras-chave: diabete tipo 2, representação social, mulher, adesão.

\section{Introducción}

La diabetes tipo 2, como enfermedad crónica, además de los efectos sociales y económicos que tiene para un país, también afecta la vida de la persona que la padece (1), en específico algunas mujeres, quienes en su esfuerzo por cumplir con sus múltiples tareas domésticas dejan su bienestar en el último lugar dentro de su lista de prioridades, incrementando el riesgo de sufrir las complicaciones generales del padecimiento y los problemas de salud reproductiva y sexual (2), que las llevan, en corto plazo, a una vida deteriorada y a un desenlace fatal.

Los anteriores factores pueden influir en la adherencia terapéutica, la cual —intencionada o no- es un problema complejo (3). Sin embargo, la adherencia terapéutica en mujeres de 25 a 49 años de edad con diabetes tipo 2 parece estar más asociada con la representación social que se construye cuando es diagnosticada, pues ello da lugar a todo un proceso de elaboración cognitiva y simbólica que orienta sus comportamientos. Esta noción de representación social intersecta lo psicológico y lo social (4), pues concierne a la manera como la mujer aprende los acontecimientos, las características del medio ambiente, la información en la cual circulan y las personas de su entorno próximo o lejano, es decir, ese conocimiento espontáneo e ingenuo denominado conocimiento natural, por oposición al conocimiento científico (5).

La teoría del núcleo central de Jean-Claude Abric (4) señala que toda representación está organizada alrededor de un núcleo central que le da estructura y lo dota de estabilidad. Está constituido por elementos considerados esenciales y los demás son periféricos. La representación social tiene tres dimensiones: 1) un núcleo central, 2) un sistema de categorización y 3) un conjunto de informaciones, actitudes y creencias. El núcleo central cumple dos funciones esenciales: 1) la generadora, mediante la cual se crea la representación social y 2) la organizadora, en la que el núcleo central determina la naturaleza de los lazos unificadores y estabilizadores de los elementos de la representación social.

Según la naturaleza del objeto y la finalidad de la situación, el núcleo central podrá tener dos dimensiones: una funcional, en la que el núcleo central lo constituyen los elementos más importantes para la realización de la tarea, y una normativa, en que intervienen directamente dimensiones socioafectivas, sociales e ideológicas. En este tipo de situaciones se puede pensar que una norma, un estereotipo o una actitud fuertemente marcada estarán en el centro de la representación. Para que un objeto sea motivo de una representación social los sistemas periféricos deberán estar directamente asociados con el objeto y originar dos tipos de representaciones sociales: 1) las autónomas, cuyo principio organizador se sitúa al nivel del objeto mismo, y 2) las no autónomas, cuyo núcleo central se sitúa fuera del objeto.

Los sistemas periféricos, cuya determinación es más individualizada y contextualizada, constituyen lo esencial del contenido de la representación. Desde este planteamiento, es necesario que al diseñar, aplicar y evaluar intervenciones de enfermería, se identifiquen los objetos que provocaron la sociogénesis de la representación social, sus elementos, el núcleo central y los sistemas periféricos, los cuales deben permear 
toda la intervención. De esta manera, se asegura la aceptación y la aplicación en la vida diaria de la persona y mejoran la relación objeto-sujeto.

\section{Metodología}

Para la investigación se llevó a cabo una revisión integrativa utilizando como guía el método prisma de artículos (6), cuyos descriptores primarios fueran representaciones sociales, diabetes tipo 2 y mujeres; mientras que el descriptor secundario fue pacientes con diabetes tipo 2, ubicados fundamentalmente en el título del artículo y que correspondieran efectiva y exclusivamente a representaciones sociales de las personas con diabetes tipo 2. Se incluyeron trabajos a texto completo tanto de carácter cuantitativo como cualitativo en inglés, español, francés y portugués. Se descartaron aquellos estudios que, a pesar de referirse al tema de representaciones sociales, se asociaban básicamente con diabetes tipo 1, incluían algún otro tipo de población o hacían referencia a algunos síndromes o patologías relacionadas con las complicaciones de la diabetes.

La búsqueda se realizó en bases de datos informatizadas: Web of Science, Medline, PubMed, SciELO, LILACS, Bireme y CUIDEN. La revisión proporcionó estudios que cumplían los siguientes criterios de inclusión: a) estudios cuantitativos y cualitativos sobre representaciones sociales de la diabetes tipo 2 en mujeres; b) escritos en inglés, español, francés o portugués, y c) investigaciones publicadas desde 1999 a 2016. Esto con el propósito de reunir conocimientos y dar respuesta a las preguntas orientadoras: ¿cuál es la representación social que la mujer tiene sobre la diabetes tipo 2? ¿Qué elementos integran el núcleo central y los sistemas periféricos de la representación social? En total se seleccionaron 120 artículos, y de estos se eligieron 45 que cumplían con los criterios de representaciones sociales de las mujeres con diabetes tipo 2 como tema central. En los trabajos escogidos se identificó cómo se abordaba el tema de acuerdo con las palabras clave y el objetivo planteado (figura 1).

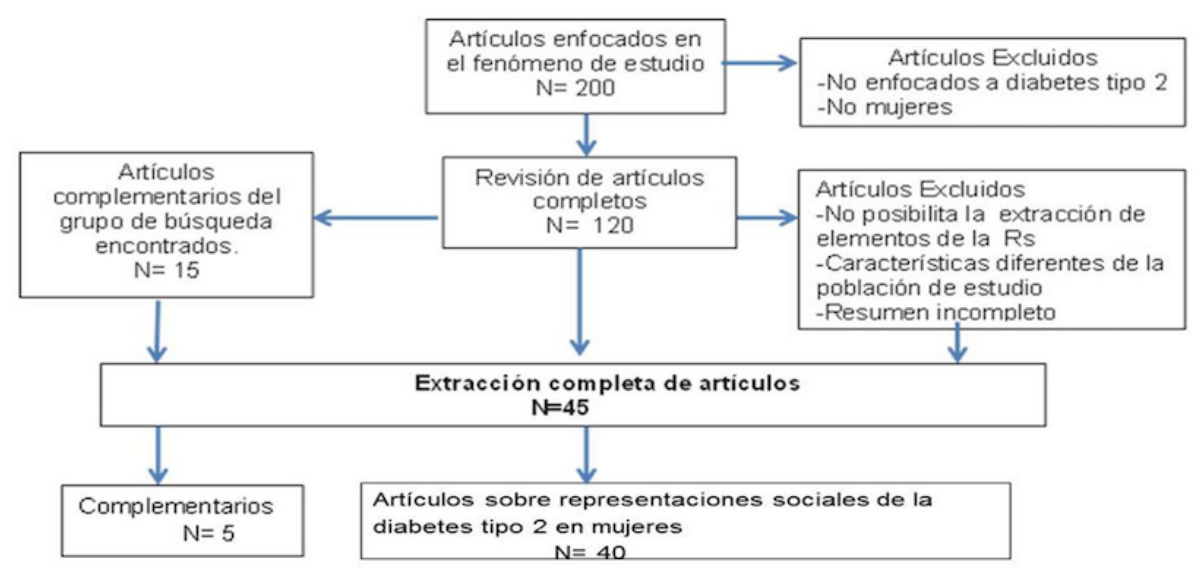

FIGURA 1.

Diagrama de flujo para la revisión de artículos

Fuente: elaboración propia.

\section{Resultados}

La recolección y análisis de contenidos mediante el método de similitudes y diferencias (7) permitió identificar la sociogénesis de la RS y sus elementos, los conceptos dominantes de padecimiento, alimentación, medicación y ejercicio, dan origen al núcleo central, el sistema periférico contextual se integró con los conceptos más comunes y los conceptos citados raramente por las mujeres quedaron integrados en el sistema periférico individual. 


\section{Sociogénesis de la representación social de la diabetes tipo 2}

La sociogénesis de la representación social está compuesta por tres fases:

Primera fase. Se da cuando nuevas prácticas sociales conflictivas se incorporan a la estructura de las representaciones sociales. En el caso de la diabetes tipo 2, las mujeres la definen como "tener azúcar en la sangre para toda la vida, enfermedad silenciosa, difícil, mala y cruel, que es cosa de otro mundo" $(8,9,10,11,12,13,14,15,16,17)$. Además de "traicionera, es voluble, lábil y sorprendente; es la enfermedad más triste del mundo. Es una enfermedad muy dura, incurable y peligrosa que erosiona la vida, presente en todo momento al no tener curación; por tanto, es la compañera de toda la vida" $(7,8,12,15,16,17,18,19,20,21)$.

Señalan que el motivo de la enfermedad fue el "estrés, un susto, un enojo, una impresión, un castigo, o designio divino; que tal vez fue por un coraje, peleas un mal echado un mal karma o malos actos que durante su vida hayan hecho" $(7,11,12,13,16,17,18,19,20,22,23,24,25,26,27,28)$. Otras causas que pudieron haber influido fue el "miedo, la emoción, la tristeza o la depresión, también, consideran que pudo haber sido por algún descuido del cuerpo, corrientes de aire o un episodio fuerte de carga emocional” (16,18,25,26,29). Pudo haber sido el "estilo de vida, consumo excesivo de alcohol, malos hábitos alimenticios, la herencia, la obesidad y por fumar" $(11,12,13,20,24,25,26,30,31)$.

En estos relatos se aprecia la voz popular sobre las causas que originan la enfermedad, las cuales pueden ser correctas o incorrectas. Esto probablemente predisponga la norma para la acción hacia el tratamiento.

Segunda fase. Se da cuando un acontecimiento perturbador se hace relevante. En el caso de la diabetes tipo 2, se encontraron transformaciones "brutales". El padecimiento irrumpió en la vida social de las personas y afectó seriamente el marco de referencia. Cuando "a mí me dijeron que tenía la diabetes, fue como una bofetada. Alteró mi vida en lo físico, social, emocional y laboral. Afectó mi estabilidad física y emocional y obligó a reorganizar la rutina. Continuamente se debe buscar, evaluar y elegir información que ayude a seguir el régimen prescrito de la dieta, medicalización y ejercicio para evitar verse disminuido y discapacitado" $(5,7,9,11,16,18,23,25,31,32,33)$. Este acontecimiento dio inicio a todo un proceso de intercambio comunicativo de creencias, informaciones, opiniones, además de comportamientos sociales con otras personas y su contexto.

Tercera fase. La representación se transforma y da lugar a ajustes en la identidad social del grupo. Para las mujeres cuidado significa "acciones que les ayudan a vivir mejor, mantener el nivel de azúcar y estabilizarse para no terminar tan rápido”. Al aceptar que padecen la enfermedad, se conforman y acostumbran, lo toman a bien y crean el compromiso activo con la enfermedad, hacen frente a la situación, queriendo estar bien y controladas. "Se dan ánimo y consuelo que les mantiene una visión positiva, dando sentido y significado a sus acciones" (5,7,9,11,16,18,21,23,28,32,34,35,36,37). Mantienen la creencia de que "sí se puede ganarle a la enfermedad y se cuidan para una mejor calidad de vida, asumiendo una actitud activa, de alegría, de amor a la vida, pidiéndole al cuerpo día a día que resista, resista y no la traicione, porque estar preparado elimina el medio y saben que cuidándose durarán más" $(9,11,16,25,28,32,33,37,38)$.

Sin embargo, algunas mujeres no alcanzan a dar identidad social a la diabetes y no tienen "apego a la alimentación, medicación y actividad física, convirtiéndose en receptoras y objetos pasivos, llevan vida sedentaria y transgresión dietética, ni toman ni hacen la dieta correcta, consumen bebidas y alimentos que causan enfermedad, no reconocen beneficios de la medicalización, argumentan que tantos cambios no lo pueden soportar y no harán lo que les dicen, ni modificarán, ni harán nada y ni tomarán nada" $(9,10,11,17,18,25,26,32,37,38)$. Ellas requieren disponer de las experiencias, acciones y conocimientos, desarrollados por la comunidad (figura 2). 


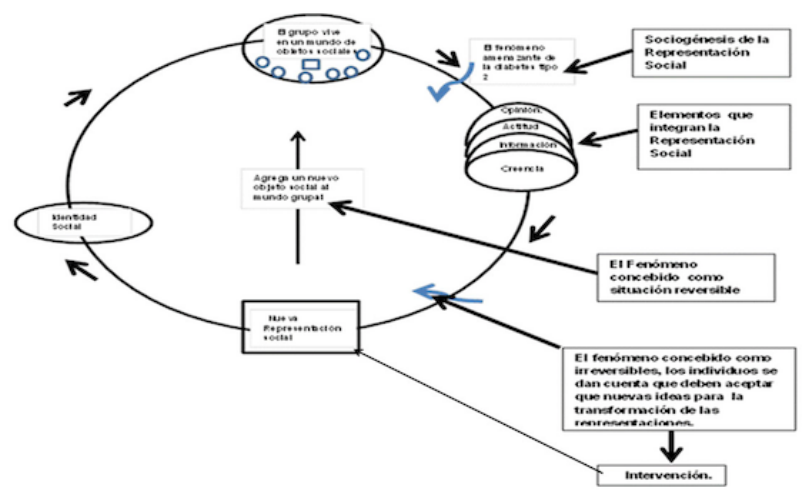

FIGURA 2.

Sociogénesis de la representación social de la diabetes mellitus tipo 2 Fuente: elaboración propia.

\section{Elementos de la representación social}

A continuación, se describen los elementos que integran la representación social que tienen las mujeres sobre la diabetes tipo 2: creencias, opinión hacia el profesional de la salud, información y actitud.

\section{Creencias}

Las mujeres tienen la creencia que la medicación no es necesaria, esto es, alivia una cosa, pero enferma otra, y después ya no hace efecto. Rechazan la medicación por incomodidad de tomar muchas pastillas; además, por sí solas, las pastillas no curan, tomarlas cansa y dan náuseas. Es una porquería de remedio que no vale. Lo toman solo cuando se acuerdan o lo dejan de tomar hasta por seis meses. Modifican la dosis y el horario de tratamiento. Creen que la insulina causa ceguera, genera vulnerabilidad física, ocasiona baja actividad sexual, pérdida de peso y provoca cansancio, daña al cuerpo y daña el riñón, lo que incrementa el miedo a su uso (6 ,9,10,11,17,18,24,27,34,36,39).

\section{Opinión hacia el profesional de la salud}

Las mujeres opinan que el equipo de salud considera al paciente apático, negligente y desinteresado de su padecimiento. Hay médicos malos que hasta hacen daño con lo que recetan. Solo mandan los estudios, dictan horarios, recomiendan hacer ejercicio, se enojan si dices que no tomaste el medicamento, amenazan con usar insulina si no cumples el tratamiento, son útiles y amables; pero "no te toman en cuenta al dictar dietas; son profesionales, pero distintos e impersonales" $(5,6,9,11,16,27,32,37,38)$. Hay "médicos buenos que explican bien sobre visitas y revisiones, dan confianza e información y dicen que no hay cura para la diabetes. Lo consideran una carga motivacional para sobrellevar su padecimiento $(6,23,27,33,37)$.

En específico, "la enfermera tiene un toque personal y da un aliento de vida, sus pláticas y consejos tienen mayor impacto y ayudan a vivir con la enfermedad" $(7,8,12,28,38)$. Con la medicina tradicional, la opinión es diferente: le tienen confianza, porque mejora la salud, puede controlar la diabetes y hasta curarla, no daña el cuerpo, no tiene restricción, no es costosa, se puede utilizar sola o con mezclas, se usa el wereque, nopal, tronadora, chancar, diente de león, piña y manzana, thelas, karamilla, sambol, moringa, planta de flor blanca, aloe vera, yerbabuena, hojas de kumquat, te de seda del maíz, y frutas autóctonas, entre otras $(17,18,27,29,35)$.

En cuanto a la alimentación, opinan que "afecta la convivencia, es limitada, la dieta no considera la alimentación cultural, se establece desde el punto de vista médico y nutriólogo que no toman en cuenta su contexto simbólico, social, cultural y temporal, el seguirla excluye de las actividades sociales y genera sensación de segregación, disocia el vínculo energético sobre todos los seres, suscita caos, choque y mixtificaciones, altera 
las situaciones culturales y es una meta difícil de seguir y de lograr, porque es difícil modificar la alimentación y se convierte en una lucha constante por el cambio de hábitos" $(2,7,8,16,18,19,23,28,30,35,38)$.

Además, el "dinero dificulta su seguimiento, son alimentos especiales y caros que no les gustan y no tiene buen sabor, como no se les informa sobre lo que deben y no deben comer para evitar incrementar los niveles de glucosa en sangre, hace que les de mucha hambre y no puedan comer. Opinan que el ejercicio no es necesario, con el trabajo diario es suficiente, además requiere vestimenta especial y no tienen tiempo, solo deben realizarlo las personas con pobre control metabólico u obesidad, si no están gordas no lo necesitan, tienen resistencia a practicarlo por miedo a caídas, tropiezos y por la falta de hábito para realizarlo" $(10,16,18,19,28,33,37,38,39)$.

\section{Información}

Las mujeres con diabetes solicitan información que les defina el padecimiento para perfeccionar los conocimientos, que se les enseñe a reconocer síntomas de hiperglucemia e hipoglucemia e identifiquen por qué se presentan las dolencias del cuerpo, cabeza, ojos, piernas y de otros síntomas como hambre, excreciones urinarias constantes, olvido, sudoración, sed constante y el cansancio que quita fuerza, quedando decaídos, con sueño, sin ganas de hacer nada, se sienten borrachas, con boca reseca, amarga y con hormigueo en la cara, la baja actividad sexual y bajo interés. Identifican la glucosa alterada porque sienten inquietud en todo el cuerpo, "deseos de beber mucha agua, mareos, debilidad, temblores, sensación de frío y piel de gallina, palpitaciones y nerviosismo" $(10,11,12,13,16,23,24,25,26,34,36,38,39)$.

Con la experiencia que se adquiere, se renuncia a la amenaza, y el compromiso con la enfermedad disminuye, por lo que se puede presentar riesgo de estar "inconsciente" y morir, aunque el temor no es a la muerte, sino a la forma de morir (amputado) $(7,9,18,23,26,31,34,37,38,39)$.

\section{Actitud}

En las mujeres es común encontrar el "sentimiento persistente de preocupación, miedo, tristeza, ansiedad, enojo, desesperación, angustia, rabia, temor, depresión, tensión y la ira diabética, dejándose arrastrar por el fatalismo, la incertidumbre, la vergüenza, el sufrimiento, la culpa, el abatimiento y la decepción" (7,10,11,12,20,21,22,24,25,26,28,29,33,39). La mujer se siente despreciada, rechazada, desesperanzada e insatisfecha, y la pérdida del bienestar y placer hacen que continuamente caiga en aburrimiento y soledad. Se siente condenada, vieja y excluida. Tiene una visión negativa, siente que nada vale la pena, su vida se apaga, y al verse inutilizada y sentirse rechazada, se aísla y le da por llorar $(5,7,9,10,11,12,16,21,23,24,25,32,33,34,37,38)$.

En la enfermedad, la fe se hace presente y se establece la relación con ese ser supremo a través de rezos, peticiones y promesas. Le piden que no se desarrolle la enfermedad y puedan recuperar la salud, les prolongue la vida, dé fortaleza para curarse y adaptarse a la nueva vida, vivir sin dolor y sobrellevar su enfermedad $(5,6,9,11,18,23,25,26,27)$. Los elementos de la representación social se visualizan en la figura 3. 


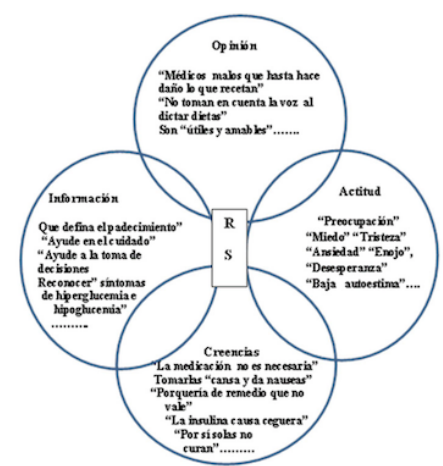

FIGURA 3.

Elementos de la representación social de la diabetes tipo Fuente: elaboración propia.

\section{Elementos que integran el núcleo central y sistemas periféricos}

Como resultado del análisis de similitud, el núcleo central quedó estructurado por los conceptos dominantes de padecimiento, alimentación, medicación y ejercicio, toda vez que fueron los conceptos más mencionados por las mujeres. Estos dan estabilidad a la representación social, y los sistemas periféricos se integraron con los conceptos más comunes como cuidados, medicina tradicional, profesionales de la salud, familia, apoyo de otros, trabajo, género femenino, servicios de salud, cambio de hábitos, conocimiento y educación, que corresponden con el sistema periférico contextual. Raramente, los conceptos citados por las mujeres quedaron integrados en el sistema periférico individual: desesperanza, curso de la enfermedad, cuidados, causa de la enfermedad, autoestima, significado común de la enfermedad, sentimientos disfuncionales, actitud, vivir con la enfermedad, negar la enfermedad, síntomas de la enfermedad, fe en Dios y esperanza como puede observarse en la figura 4.

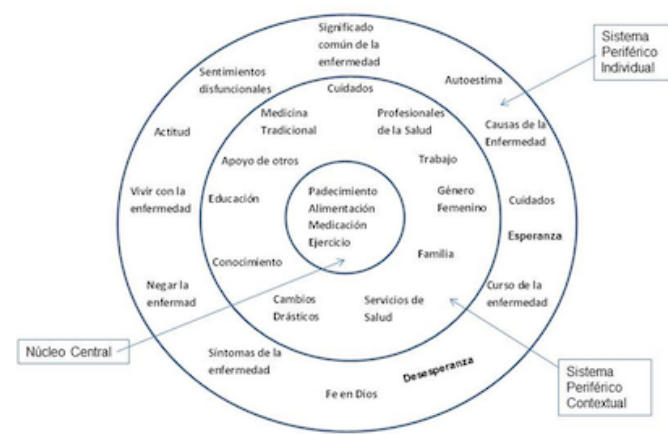

FIGURA 4.

Componentes centrales y sistemas periféricos de la representación social que construyen las mujeres sobre la diabetes tipo 2

Fuente: elaboración propia.

\section{Discusión}

Para enfermería, el cuidado es su razón de ser; por tanto, la situación de cronicidad actual le exige replantear ese cuidado desde lo cognitivo y desde lo psicológico. Diversos autores como Montalvo-Prieto et al. (39) destacan que padecer una enfermedad crónica implica sufrimiento, dolor y sentimientos de frustración y tristeza. González-Cantero et al. (40) agregan que también se presenta malestar, rechazo y enojo. Para lograr este objetivo una alternativa sería utilizar en la planeación del cuidado teorías sociales, como en este caso la del nucleó central, toda vez que ayuda a comprender los acontecimientos dramáticos y dolorosos que 
se presentan en la diabetes tipo 2 y cómo afectan de manera importante la vida social, la comunicación y la interacción social de las personas y cómo se van formando y fortaleciendo los nodos centrales con sus experiencias individuales y grupales.

Este trabajo permitió identificar que los elementos que integran el núcleo central, concordando con Torres et al. (41), son la alimentación, la medicación y el ejercicio, y que alrededor de ellos giran determinismos históricos e individuales que establecen la conducta de las mujeres. Estos determinismos pudieran estar afectando la adherencia terapéutica, la cual curiosamente está integrada por la alimentación, la medicación y el ejercicio. La teoría señala que el núcleo central es el más resistente al cambio; sin embargo, intervenciones encaminadas a modificar los sistemas periféricos impactan y modifican el núcleo central.

Los hallazgos marcan la pauta para planear intervenciones que, como señala Pisano y González (42), modifiquen los hábitos y fortalezcan la adherencia terapéutica abordando el padecimiento crónico, no solo desde la visión asistencial con un modelo médico hegemónico que, como menciona Giraudo (37), los separa de su contexto social cultural e histórico, sino desde la mirada del cuidado integral, que toma en cuenta la voz de la persona que vive y sufre la enfermedad.

\section{Conclusión}

En síntesis, la enfermedad crónica, lejos de ser solo síntomas y signos, es un evento que modifica la vida de la mujer, su familia y su entorno. Esto hace necesario que, al planear el cuidado de enfermería, se tomen en cuenta las necesidades en específico de las mujeres tanto en lo cognitivo como en lo psicológico; pensar no solo en el modelo biomédico, sino en todos los saberes y formas de mejor atención con las que se busca prevenir, tratar, controlar, aliviar o curar un padecimiento. Por tanto, este artículo de revisión intenta aportar una forma diferente de enfrentar el problema de la diabetes tipo 2, en el desarrollo de programas de intervención enfocados en modificar la representación social de la enfermedad y la adherencia terapéutica, a fin de mejorar la calidad de vida de las mujeres.

\section{Limitaciones}

El estudio se limitó a explorar la representación social que tienen las mujeres sobre la diabetes tipo 2, toda vez que se requieren programas de intervención que en su diseño tomen en cuenta el sentido común de lo que piensa y dice la gente que padece la enfermedad, utilizando teorías como la del núcleo central para favorecer el cuidado de enfermería.

\section{Referencias}

1. International Diabetes Federation [internet]. Madrid: Federación Internacional de Diabetes; c1950 [citada 15 de mayo de 2017]. Disponible en: https://www.idf.org.

2. Secretaría de Salud. Norma Oficial Mexicana para la prevención, tratamiento y control de la diabetes mellitus [internet]. 2012 [NOM-015-SSA2-2012]. México: Secretaría; 2012.

3. Secretaría de Salud. Encuesta Nacional de Salud y Nutrición de Medio Camino 2016 [internet]. México; c2016 [citada 20 de marzo de 2017]. Disponible en: https://www.promocion.salud.gob.mx/dgps/descargas1/doctos_ 2016/ensanut_mc_2016-310oct.pdf.

4. Abric J-C. Prácticas sociales y representaciones. México: Coyoacán; 2001.

5. Rateau P, Lo Monaco G. La teoría de las representaciones sociales: orientaciones conceptuales, campos de aplicaciones y métodos. Revista CES Psicología. 2013;6(I):22-42. 
6. Brian HF, Catalá-López DM. La extensión de la declaración PRISMA para revisiones sistemáticas que incorporan metaanálisis en red: PRISMA-NMA. Rev Med Clín. 16 Sep 2016 [citado 15 de mayo de 2017];147(6). https: //www.doi.org/10.1016/j.medcle.2016.10.003.

7. Lucie L. L'analyse de similitude pour modéliser les CHD. Revista JADT [internet]. 2016 [citado 20 de marzo de 2017]. Disponible en: https://www.lexicometrica.univ-paris3.fr/jadt/jadt2016/01-ACTES/83440/83440.pd f.

8. Paz RC, Sandoval J, Falque ML, Stepenka V, Maestre GE, Rivas Y, et al. La construcción social acerca de la diabetes y la prediabetes en personas que acuden a un centro de salud en Maracaibo Venezuela. Revista Internacional de Salud, Bienestar y Sociedad. 2014;1(2):59-68.

9. Marques TR, Dumêt FJ, Andrade CE, Almeida SS. Structure of social representations of people's accession to diabetes treatment. Journal of Nursing UFPE On Line. 2014 [citado 15 de mayo de 2017];8(10). https://www .doi.org/10.5205/reuol.6039-55477-1-ED.0810201420.

10. Madia MA, Prado TC, Bizetti PE, Madia MA, Savian UN, Pagotto P. A comparative study of social representations of diabetes mellitus and diabetic foot. Cad Saude Publica. 2013 [citado 15 de enero de 2017];29(12). https:// www.dx.doi.org/10.1590/0102-311X00006613.

11. Hatcher E, Whittemore R. Hispanic adults' beliefs about type 2 diabetes: clinical implications. J Am Acad Nurse Pract. 2007;19(10). https://www.doi.org/10.1111/j.1745-7599.2007.00255.x.

12. García-Reza C, Alvirde-Vara R, Landeros-López M, Solano-Solano G, Medina-Castro ME. Resistencia frente a la enfermedad: relato de un cuerpo dañado por la diabetes. Aquichan. 2014;14(4). https://www.doi.org/10.5294 /aqui.2014.14.4.4

13. Gilles BB, Courvoisier N, Clémence. Inhabitants' and professionals' social representations of health determinants in a disadvantaged urban area in France: A qualitative analysis. Revue d'Epidemiologie et de Santé Publique. 2014;62(1):5-14. https://www.doi.org/10.1016/j.respe.2013.08.003.

14. Machado HB, Koelln CR. Crenças sobre saúde: influência nas atitudes de portadores de doenças crônicodegenerativas em relação ao cuidado à saúde. Revista Baiana de Enfermagem. 2009;22(23). https://www.dx.do i.org/10.18471/rbe.v22i1.4992.

15. Siqueira PD, Franco LJ, Dos Santos MA. Sentimentos de mulheres após o diagnóstico de diabetes tipo 2. Rev Latino-Am Enfermagem. 2008;16(1). https://www.dx.doi.org/10.1590/S0104-11692008000100016.

16. Heather LS, Mullan-Jensen CB, Reach G, Kovacs KB, Piana N, Vallis M. et al. Personal accounts of the negative and adaptive psychosocial experiences of people with diabetes in the second Diabetes Attitudes, Wishes and Needs (DAWN2) study. Diabetes Care. 2014;37(9). https://www.doi.org/10.2337/dc13-2536.

17. Page-Pliego JT. Subjetividades sobre la causalidad de diabetes mellitus entre seis tzeltales de la cabecera municipal de Tenejapa, Chiapas. Limina R [internet]. 2015;13(2). Disponible en: https://www.scielo.org.mx/scielo.php? script $=$ sci_arttext\&pid=S1665-80272015000200007.

18. Moldovan R. An analysis of the impact of irrational Beliefs and illness representation in predicting distress in cancer and type II diabetes patients. Cogn Brain Behav [internet]. 2009;13(2):179-93. Disponible en: https://www.p sycnet.apa.org/record/2009-24911-005.

19. Lerin Piñón S, Juárez C, Reartes D. Creencias de indígenas chiapanecos en torno a la diabetes y posibilidades de atención intercultural. Revista Salud Problema [internet]. 2015;9(17):27-41. Disponible en: https://www.bidi .xoc.uam.mx/MostrarPDF.php.

20. Figueroa ME Moreno RB. Rescatar la vivencia de padecer diabetes en personas de ciudad Nezahualcóyotl, para contribuir a una teoría por sus protagonistas. Estudios de Antropología Biológica [internet]. 2007. [citada 15 de febrero de 2016];13(2). Disponible en: https://www.journals.unam.mx/index.php/eab/article/view/26423.

21. Amarasekara AA, Fongkaew W, Turale S, Wimalasekara SW, Chanprasit. An ethnographic study of diabetes health beliefs and practices in Sri Lankan adults. Int Rev Nurs. 2014;61(4). https://www.doi.org/10.1111/inr.12136 en 10.1111/inr.12136. 
22. Gomersalla T, Madilla A, Summers KM. Getting one's thoughts straight: A dialogical analysis of women's accounts of poorly controlled type 2 diabetes. Psychol Health. 2012;27(3). https://www.doi.org/10.1080/08870446.20 11.583649 .

23. Bautista F. Prevención intercultural de la diabetes tipo II en pueblo pemon kamarakoto. Boletín Antropológico [internet]. 2014 [citada 30 de agosto de 2016];32(87):28-44. Disponible en: https://www.redalyc.org/articulo .oa? $\mathrm{id}=71232464003$.

24. Scollan-Koliopoulos M, O'Connell KA, Walker E. Assessing legacies of diabetes: recollections of family members' illness representations and outcomes. J Theor Const Test. 2006;9:40-8.

25. Mercado-Martínez FJ, Robles SL, Ramos NM Alcántara HE. La perspectiva de los sujetos enfermos: Reflexiones sobre pasado, presente y futuro de la experiencia del padecimiento crónico. Cad Saúde Pública [internet]. 1999 [citada 20 de enero de 2017];15(1). Disponible en: https://www.scielo.br/pdf/\%0D/csp/v15n1/0047.pdf.

26. Luchetti RJ, Zanetti ML, dos Santos MA, Aparecida MT, Sousa VD, de Sousa TC. Conocimiento y actitudes: componentes para la educación en diabetes. Rev Latino-Am Enfermagem Jul 2009;17(4). https://www.dx.doi. org/10.1590/S0104-11692009000400006.

27. García Cedillo I, Morales Antúnez B. Eficacia de la entrevista motivacional para promover la adherencia terapéutica en pacientes con diabetes mellitus tipo 2. Univ Psychol. 2015;14(2):511-22. https://www.doi.org/10.11144/J averiana.upsy 14-2.eemp.

28. García A, Javier E, Salcedo RA, López CB. Una aproximación al conocimiento cultural de la diabetes mellitus tipo 2 en el occidente de México. Desacatos [internet]. 2006 [citada 20 septiembre 2016];21. Disponible en: https: //www.redalyc.org/pdf/139/13902107.pdf.

29. Viera da Silva DM, Hegadoren K, Lasiuk G. La perspectiva de ama de casa brasileña sobre la vida con diabetes mellitus tipo 2. Rev Latino-Am Enfermagem [internet]. 2012 [citada 30 de septiembre de 2016];20(3). Disponible en: https://www.scielo.br/pdf/rlae/v20n3/es_a07v20n3.pdf

30. Tan PS-X, Chen H-C, Taylor BJ, Hegney DG. Exploring the meaning of hypoglycaemia to community-dwelling Singaporean Chinese adults living with type 2 diabetes mellitus. Int J Nurs Pract. 2012;20(3).

31. Livier M. Grupos de autoayuda para diabéticos en contexto rural: la participación de los enfermos. Dimensión Antropológica [internet]. 2012 [citada 7 de febrero de 2017];19(54). Disponible en: https://www.dimensiona ntropologica.inah.gob.mx/?p=7681

32. Martínez-Herrera E, Moreno-Mattar O, Dover HV. El significado del capital social "individual" en diabéticos receptores de cuidado en un contexto urbano colombiano. Cad Saúde Pública. 2015;31(4). https://www.dx.do i.org/10.1590/0102-311X00167113.

33. Alves AJ, Castro VC, Ribeiro NH, Faustino DE. La incertidumbre en la enfermedad y la motivación para el tratamiento en diabéticos tipo 2. Rev Latino-Am Enfermagem. 2007;15(4). https://www.dx.doi.org/10.1590/ S0104-11692007000400009.

34. León P, Landgrave GC, Patiño DC. Representación social del apoyo familiar al diabético en usuarios de una unidad de medicina familiar en Chalco, Estado de México. Medwave. 2014;14(7). https://www.10.5867/medwave.20 14.07.6011

35. Mc JS, Bishop FL, Moss-Morris R. The chicken and egg thing': Cognitive representations and self-management of multimorbidity in people with diabetes and depression. J Health Psychol. 2013;28(1). https://www.dx.doi.org /10.1080/08870446.2012.716438.

36. Torres RM, Fernandez JD, Cruz EA. Structure of social representations of people's accession to diabetes treatment. J Nurs UFPE. 2014;8(10). https://www.doi.org/10.5205/reuol.6039-55477-1-ED.0810201420.

37. Giraudo N. Una mirada social del proceso salud/enfermedad/atención de la enfermedad crónica: un aporte desde el enfoque relacional y las representaciones sociales. Archivos de Medicina Familiar y General [internet]. 2015 [citada 20 de marzo de 2017];12(2). Disponible en: https://www.archivos.famfyg.org/revista/index.php/amfy $\mathrm{g} /$ article/view/199.

38. Ofman SD. Aproximaciones al estudio de las representaciones sociales, de la salud y enfermedad: el caso de la diabetes mellitus. Psicología y Psicopedagogía. 2012;27:34-42. 
39. Montalvo-Prieto AB, Cabrera-Nanclares B, Quiñones-Arrieta S. Enfermedad crónica y sufrimiento: revisión de literatura. Aquichan [internet]. 2012 [citado 18 de septiembre de 2017];12(2). Disponible en: https://www.re dalyc.org/articulo.oa?id=74124103005.

40. González-Cantero JO, OropezaT. Intervenciones cognitivo conductuales para diabéticos en México. Revista Salud Mental. 2016;39(2). https://www.doi.org/10.17711/SM.0185-3325.2016.006.

41. Torres RM, Fernández JD, Cruz EA. Structure of social representations of people's accession to diabetes treatment. J Nurs UFPE. 2014;8(10). https://www.doi.org/10.5205/1981-8963-v8i10a10073p3412-3419-2014.

42. Pisano GM, González PA. La modificación de los hábitos y la adherencia terapéutica, clave para el control de la enfermedad crónica. Enferm Clin. 2013;24(1). https://www.doi.org/10.1016/j.enfcli.2013.10.006.

\section{Notas}

*Artículo original de investigación

\section{Licencia Creative Commons CC BY 4.0}

Cómo citar: Landa Rivera RA, Castillo Arcos LC, Padilla Raygoza N. Representación social de la diabetes tipo 2 en mujeres bajo la teoría del núcleo central. Investig Enferm Imagen Desarr. 2019;21(1). https:// www.doi.org/10.11144/Javeriana.ie21-1.rsdm 